\title{
Large Piezoelectric Response in Hybrid Rare-Earth Double Perovskite Relaxor Ferroelectrics
}

\author{
Chao Shi, Jia-Jun Ma, Jia-Ying Jiang, Miao-Miao Hua, Qi Xu, Hui Yu, Yi Zhang*, Heng-Yun Ye* \\ Chaotic Matter Science Research Center, Department of Materials, Metallurgy and Chemistry, Jiangxi University of \\ Science and Technology, Ganzhou 341000, P. R. China
}

Property Measurements. Differential scanning calorimetry (DSC) measurements were carried out on a netzsch differential scanning calorimeter (polyma) under nitrogen at atmospheric pressure, with a heating/cooling rate of $10 \mathrm{~K} / \mathrm{min}$. For dielectric measurements, single-crystal plates with well-defined directions were used. The directions of the crystals were determined on a Rigaku diffractometer (synergy). Silver conducting paste deposited on the plate surfaces was used as electrodes. Pyroelectric property was measured with an electrometer/high resistance meter (keithley 6517B) with the cooling rate of $10 \mathrm{~K} / \mathrm{min}$. A Tonghui TH2828A impedance analyzer was used to measure the complex dielectric constants. The $P-E$ hysteresis loops were determined from current density-electric field (J-V) curves using the double-wave method [Nat. Commun. 2014, 5, 3279; J. Phys. Soc. Jpn. 2008, 77, 064706].

The piezoelectric constants $d_{33}$ were measured with a quasi-static $d_{33}$ piezoelectric instrument (ZJ-3AN, Institute of Acoustics, Chinese Academy of Sciences). The single-crystal plates were carefully mounted between the two electrodes of the piezoelectric instrument, and were kept perpendicular to the electrodes so that the shear force was avoided. The frequency dependence was measured on $d_{33}$ PiezoMeter Systems (Piezotest Pte. Ltd.).

Nanoscale polarization imaging, local switching spectroscopy and resonance peaks were carried out using a resonant-enhanced piezoresponse force microscopy (MFP-3D, Asylum Research). Conductive Pt/Ir-coated silicon probes (EFM-50, Nanoworld) were used. The measurement was carried out on the thin crystals, which were prepared as follows. Firstly, commercial ITO-coated glass substrate was ultrasonic cleaned in ethanol for 20 minutes. A drop of aqueous solution of $(\mathrm{RM} 3 \mathrm{HQ})_{2} \mathrm{RbLa}\left(\mathrm{NO}_{3}\right)_{6} /(\mathrm{RM} 3 \mathrm{HQ})_{2} \mathrm{NH}_{4} \mathrm{La}\left(\mathrm{NO}_{3}\right)_{6}(20 \mathrm{mg} / \mathrm{ml})$ was carefully spread on a freshly 
cleaned ITO-coated glass. The slow evaporation gave the thin film consisting of small thin crystals, whose shape is similar to the large crystals.

Crystal structure determination. Powder X-ray diffraction (PXRD) data were obtained on a Rigaku D/MAX 2000 PC X-ray diffractometer. Single-crystal diffraction data were collected on a Rigaku synergy diffractometer using $\operatorname{Mo}_{\alpha} \quad(\lambda=0.71073 \AA)$ radiation from a graphite monochromator. All the structures at different phases of $(\mathrm{RM} 3 \mathrm{HQ})_{2} \mathrm{RbLa}\left(\mathrm{NO}_{3}\right)_{6} /(\mathrm{RM} 3 \mathrm{HQ})_{2} \mathrm{NH}_{4} \mathrm{La}\left(\mathrm{NO}_{3}\right)_{6}$ are suggested to have the space group $P 2_{1} 3$. However, the structures below ferroelectric transition temperatures assume the space group $R 3$ based on the physical properties. The crystal structures were resolved by the direct method and then refined by full-matrix least-square method based on $F^{2}$ using the SHELXTL program (G.M. Sheldrick, Crystal structure refinement with SHELXL, Acta Cryst., 2015, C71, 3-8). The structures contain totally disordered organic cations. They were not modelled as the chemical senses. This led to the relatively high $R 1$ values.

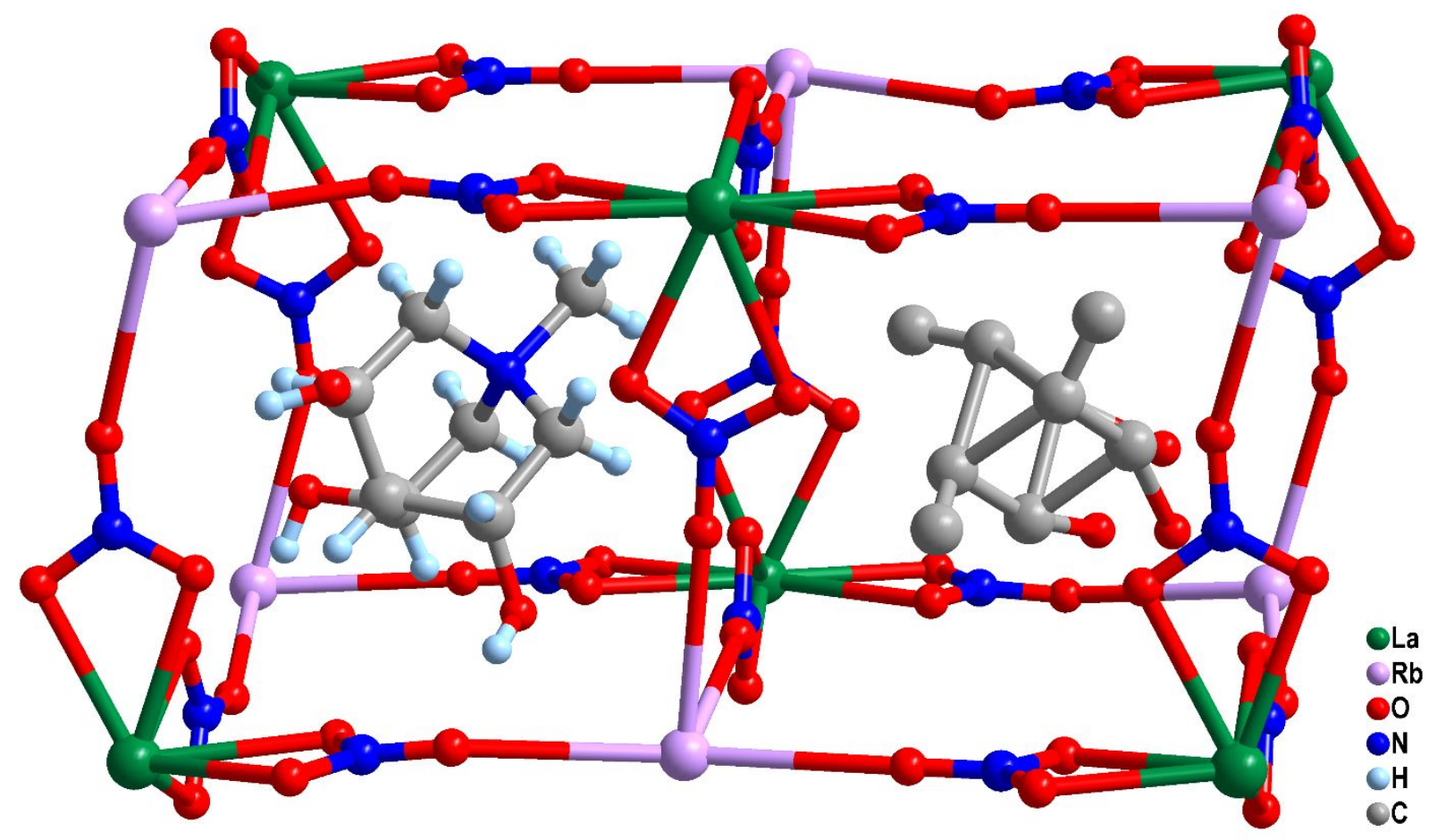

Figure $\mathrm{S} 1$. The double perovskite structure of $(\mathrm{RM} 3 \mathrm{HQ})_{2} \mathrm{RbLa}\left(\mathrm{NO}_{3}\right)_{6}(\mathbf{1})$. 

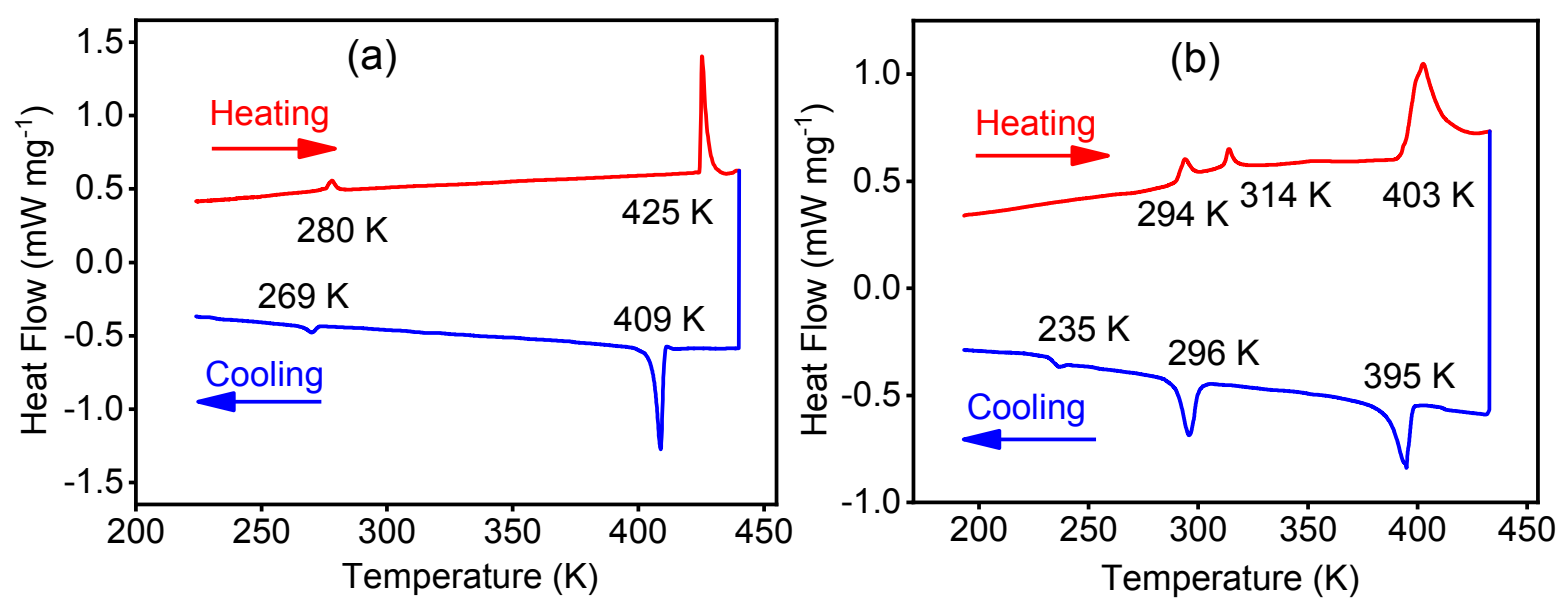

Figure S2. DSC curves for (a) 1 and (b) $(\mathrm{RM} 3 \mathrm{HQ})_{2} \mathrm{NH}_{4} \mathrm{La}\left(\mathrm{NO}_{3}\right)_{6}$ (2), revealing multiple phase transitions respectively.

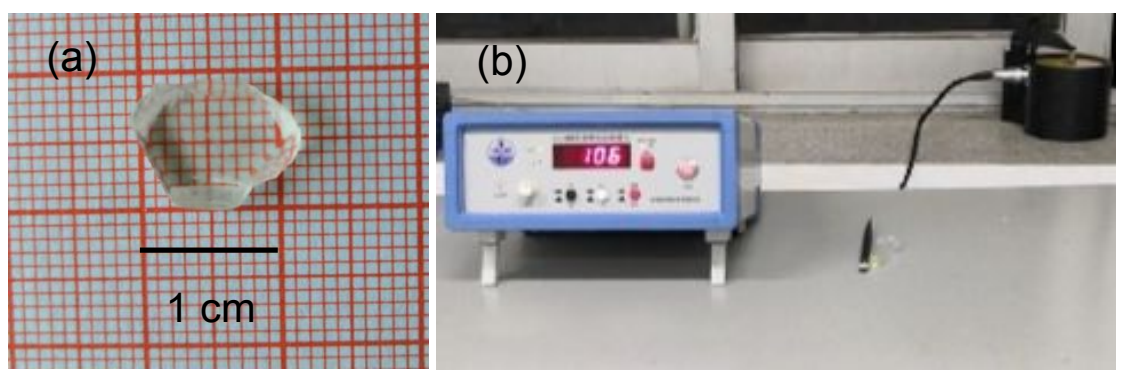

Figure S3. Piezoelectricity in 1. (a) A picture of the large crystal of $\mathbf{1}$. The largest natural face is (111)-face of the $P 2_{1} 3$ phase. (b) Recording the piezoelectric constant $d_{33}$ of a plate of 1 perpendicular to the largest natural face. 
(a)
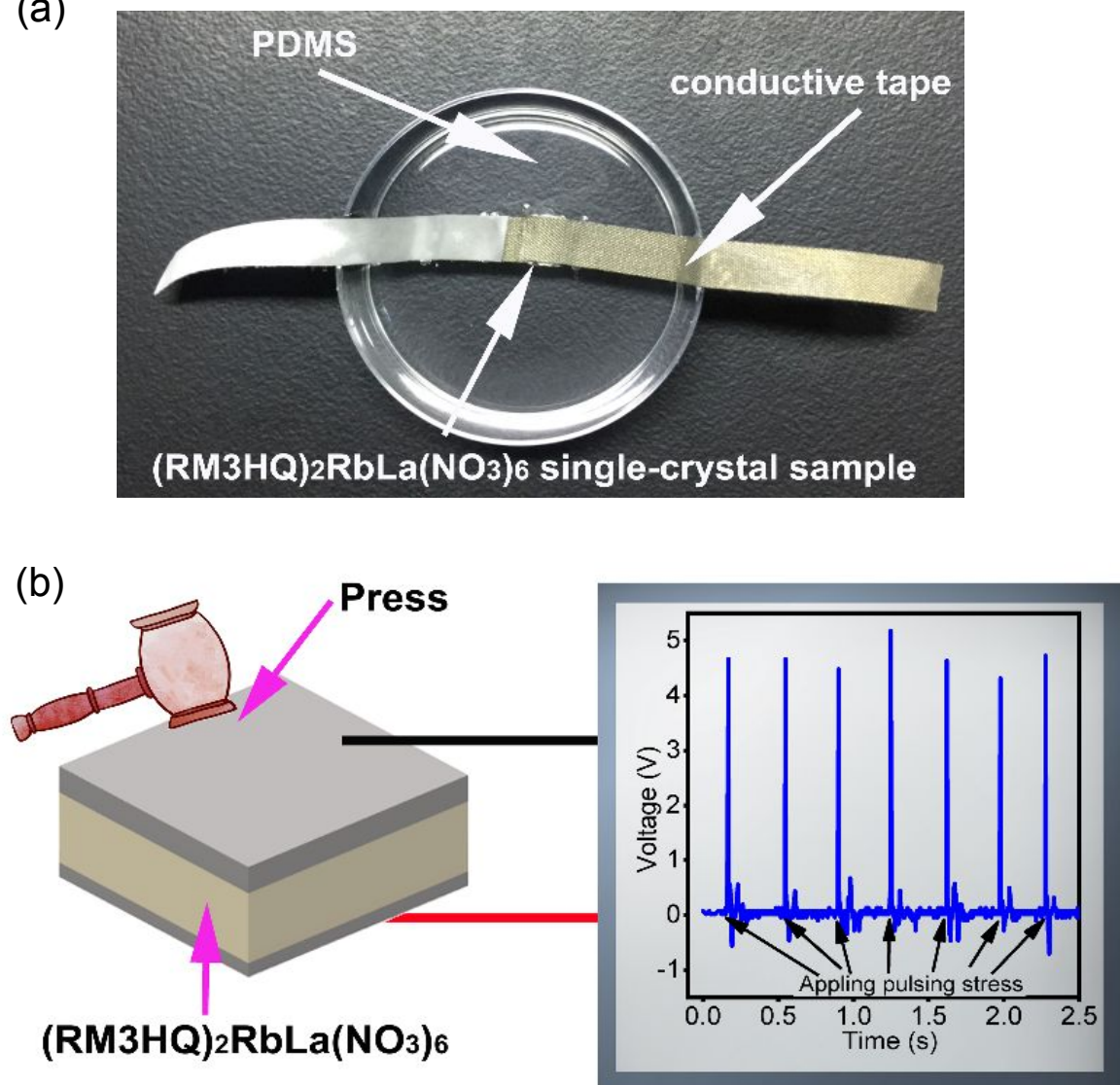

Figure S4. (a) Fabrication of a piezoelectric device for testing piezoelectric voltage of 1. (b) Schematic diagram of piezoelectric voltage examination.

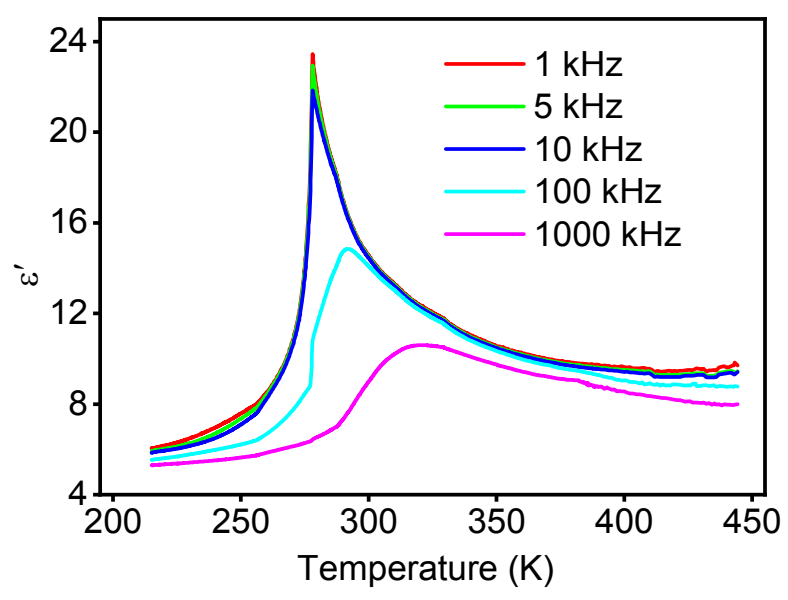

Figure S5. Dielectric curves for $\mathbf{1}$ in the cooling run, revealing that only the phase transition at around $T_{\mathrm{m}}=278 \mathrm{~K}$ is dielectric-active. 

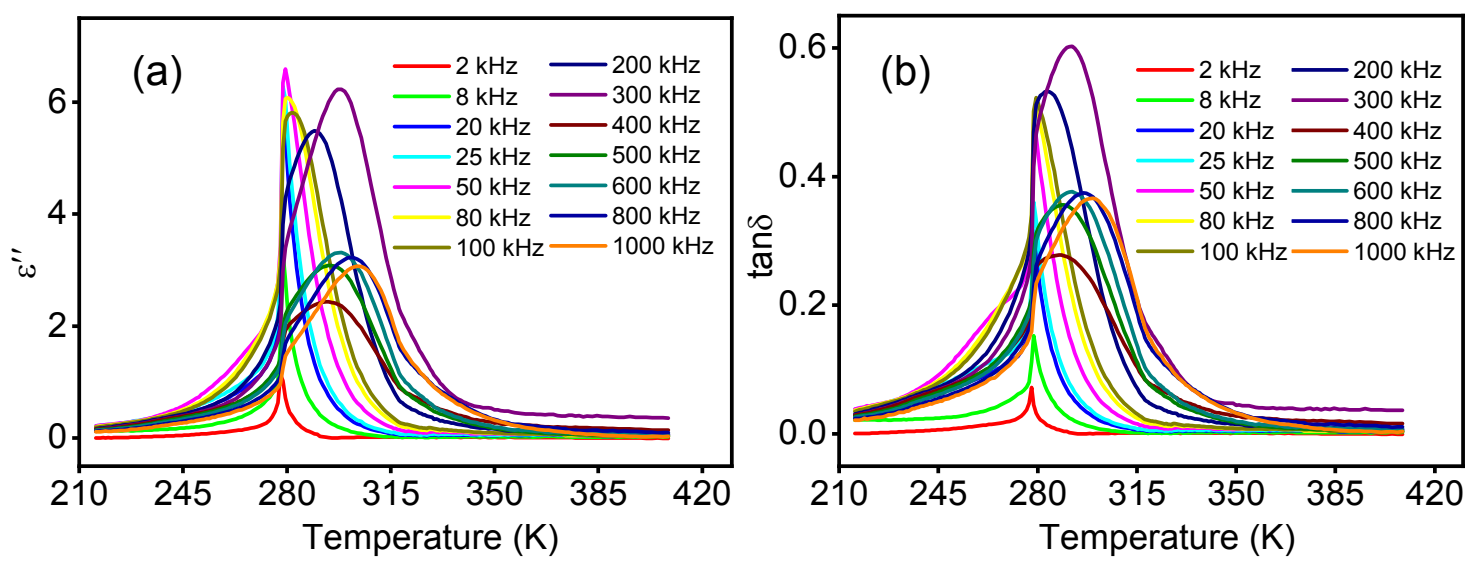

Figure S6. Dielectric curves for $\mathbf{1}$ in the heating run. (a) Temperature-variable $\varepsilon^{\prime \prime}$ at different frequency, showing the dispersive feature. (b) Temperature-variable dielectric loss.

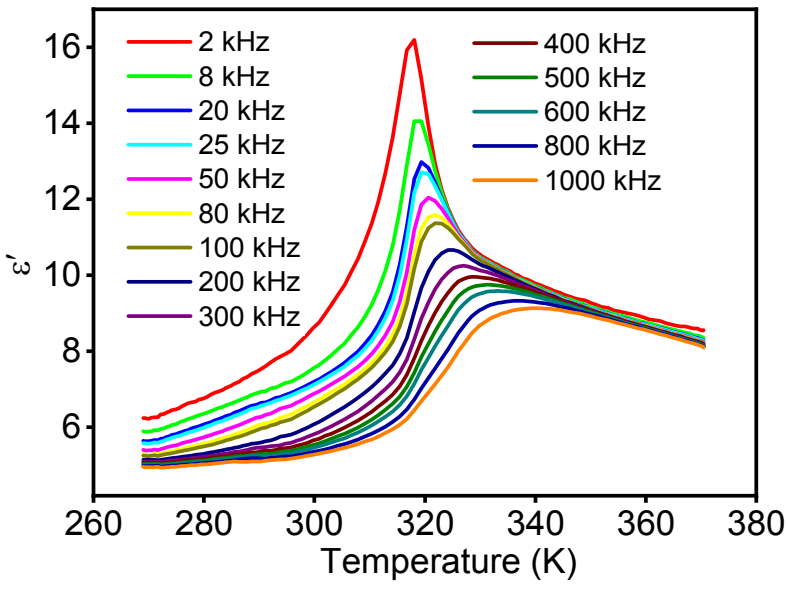

Figure S7. Dielectric curves for $\mathbf{2}$ in the heating run, showing the dispersive feature. 


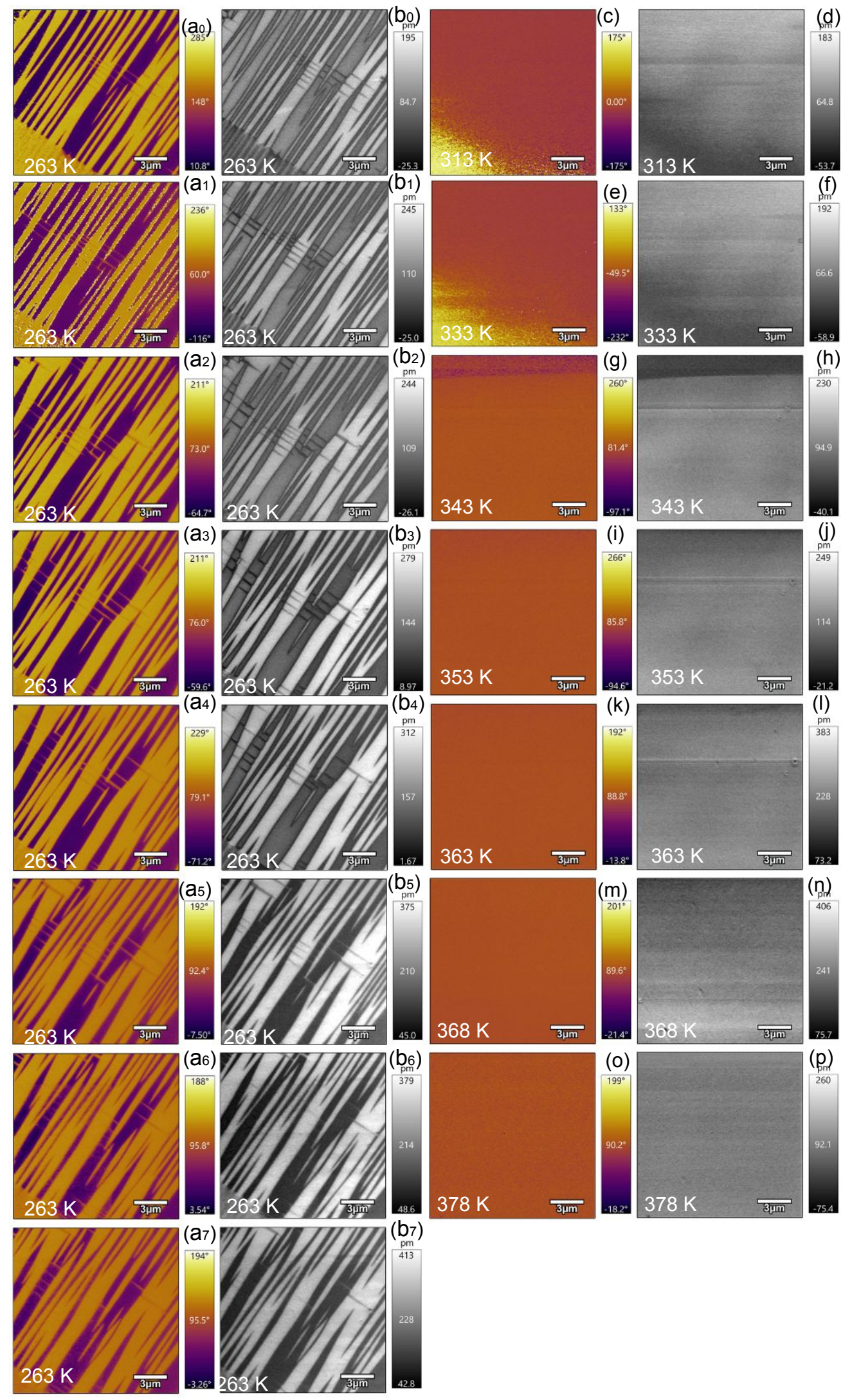

Figure S8. Evolution of the domain structure of a sample of 1 in a heating and cooling run. $\left(a_{0}, b_{0}\right)$ Vertical PFM phase and amplitude images at $263 \mathrm{~K}$, respectively. (c-- $\left.\mathrm{b}_{7}\right)$ Evolution of the domain structure from $\left(a_{0}, b_{0}\right)$ at $263 \mathrm{~K} \rightarrow(\mathrm{c}, \mathrm{d})$ at $313 \mathrm{~K} \rightarrow\left(\mathrm{a}_{1}, \mathrm{~b}_{1}\right)$ at $263 \mathrm{~K} \rightarrow(\mathrm{e}, \mathrm{f})$ at $333 \mathrm{~K} \rightarrow\left(\mathrm{a}_{2}, \mathrm{~b}_{2}\right)$ at $263 \mathrm{~K} \rightarrow(\mathrm{g}, \mathrm{h})$ at $343 \mathrm{~K} \rightarrow\left(\mathrm{a}_{3}, \mathrm{~b}_{3}\right)$ at $263 \mathrm{~K} \rightarrow(\mathrm{i}, \mathrm{j})$ at $353 \mathrm{~K} \rightarrow\left(\mathrm{a}_{4}, \mathrm{~b}_{4}\right)$ at $263 \mathrm{~K} \rightarrow(\mathrm{k}, \mathrm{l})$ at $363 \mathrm{~K} \rightarrow\left(\mathrm{a}_{5}\right.$, 
$\left.\mathrm{b}_{5}\right)$ at $263 \mathrm{~K} \rightarrow(\mathrm{m}, \mathrm{n})$ at $368 \mathrm{~K} \rightarrow\left(\mathrm{a}_{6}, \mathrm{~b}_{6}\right)$ at $263 \mathrm{~K} \rightarrow(\mathrm{o}, \mathrm{p})$ at $378 \mathrm{~K} \rightarrow\left(\mathrm{a}_{7}, \mathrm{~b}_{7}\right)$ at $263 \mathrm{~K}$. The results reveal that the paraelectric phase at $378 \mathrm{~K}$ still contain polar regions. 

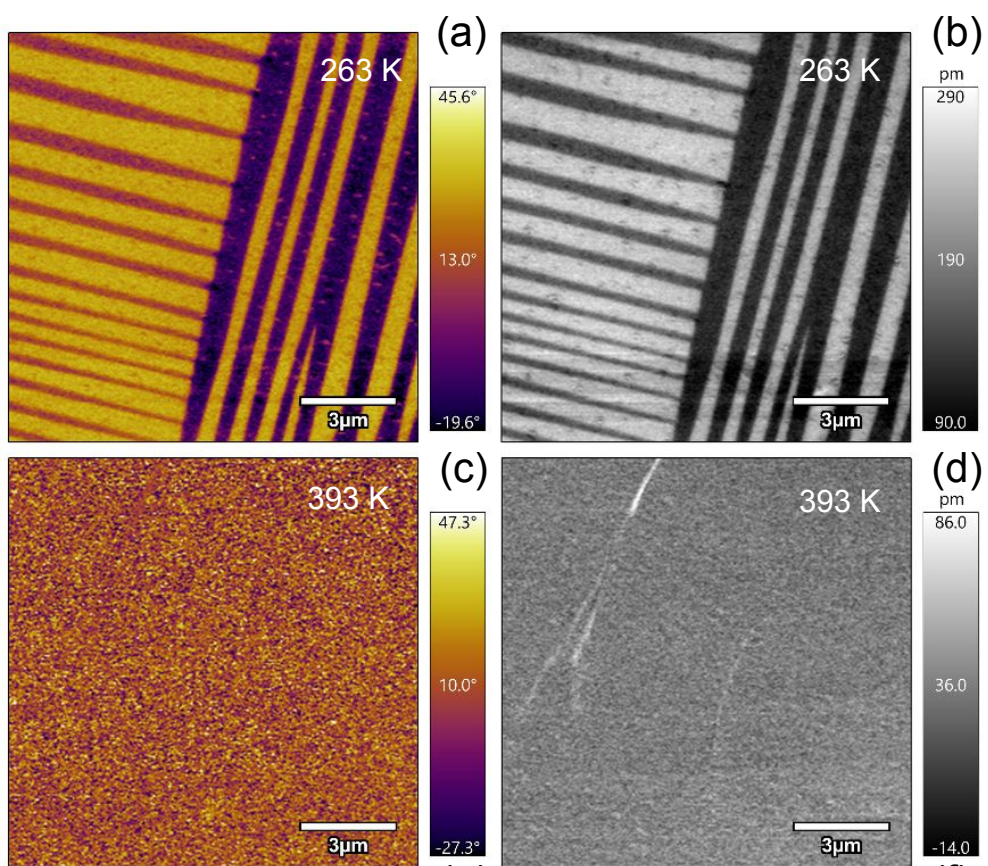

(c)
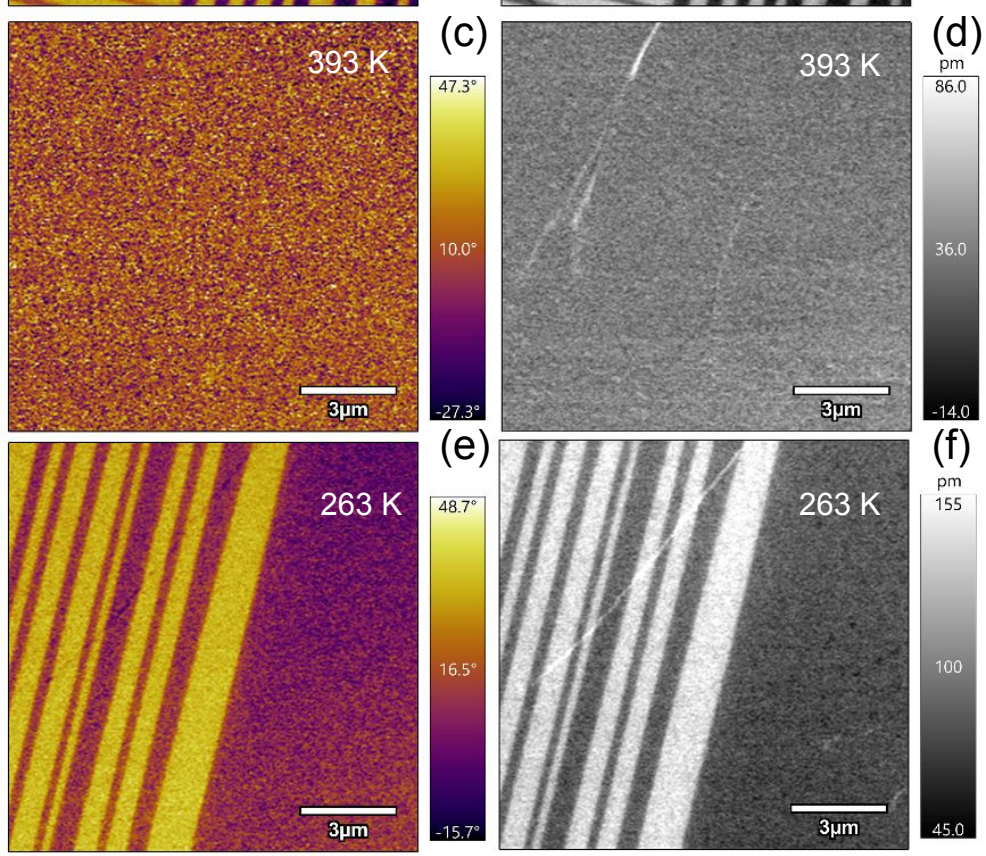

Figure S9. Evolution of the domain structure of a sample of 1 in a heating and cooling run. $(a, b)$ Vertical PFM phase and amplitude images at $263 \mathrm{~K}$, respectively. (c-f) Evolution of the domain structure from $(\mathrm{a}, \mathrm{b})$ at $263 \mathrm{~K} \rightarrow(\mathrm{c}, \mathrm{d})$ at $393 \mathrm{~K} \rightarrow(\mathrm{e}, \mathrm{f})$ at $263 \mathrm{~K}$. The results reveal that the paraelectric phase at $393 \mathrm{~K}$ contain non polar regions.
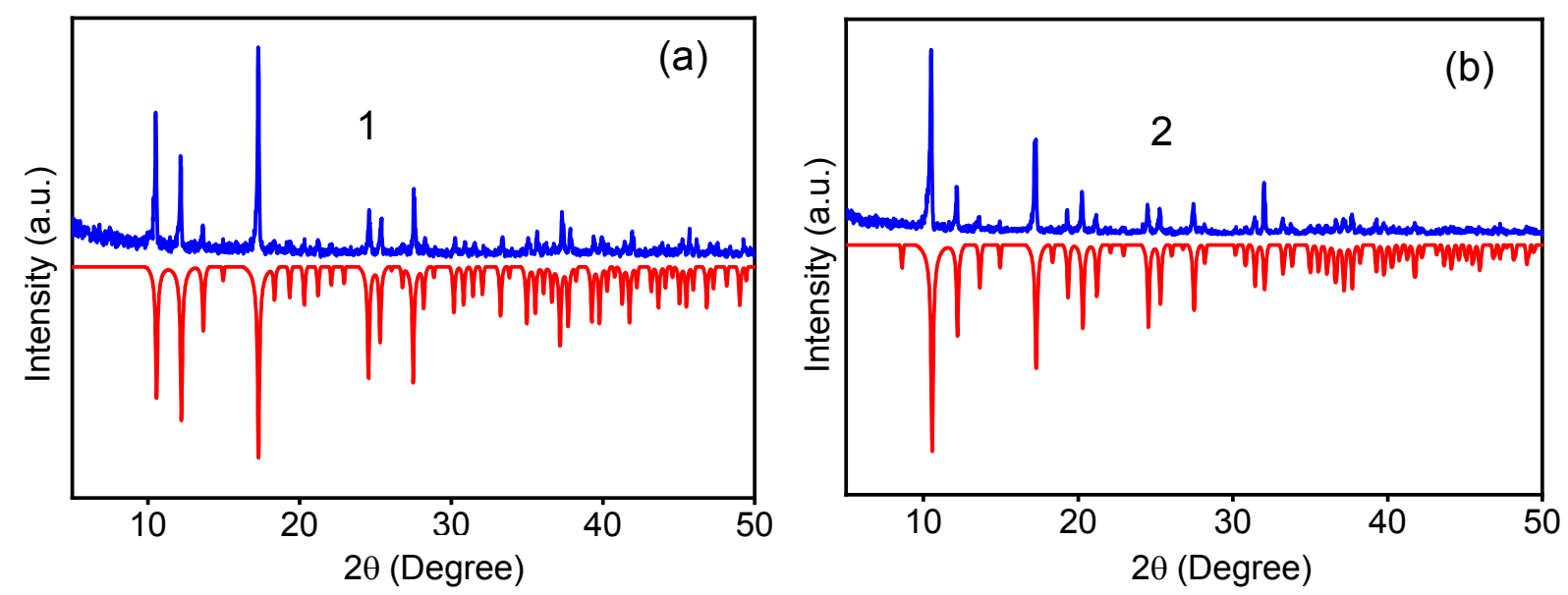

Figure S10. Patterns of the powder X-ray diffraction for (a) 1 and (b) 2. 